\title{
Imaging Electronic Structure of Carbon Nanotubes by Voltage-Contrast Scanning Electron Microscopy
}

\author{
Aravind Vijayaraghavan ${ }^{1}(\bowtie)$, Sabine Blatt ${ }^{1,2}$, Christoph Marquardt ${ }^{1,2}$, Simone Dehm ${ }^{1}$, Raghav Wahi ${ }^{1, \S}$, \\ Frank Hennrich ${ }^{1}$, and Ralph Krupke ${ }^{1}(\bowtie)$ \\ ${ }^{1}$ Institut für Nanotechnologie, Forschungszentrum Karlsruhe, D-76344 Eggenstein-Leopoldshafen, Germany \\ ${ }^{2}$ Physikalisches Institut, Universität Karlsruhe, D-76021 Karlsruhe, Germany \\ ${ }^{\S}$ Present address: Rice University, Houston, TX 77251, USA \\ Received: 30 May 2008/ Revised: 27 August 2008/Accepted: 27 August 2008 \\ CTsinghua Press and Springer-Verlag 2008. This article is published with open access at Springerlink.com
}

\begin{abstract}
We introduce voltage-contrast scanning electron microscopy (VC-SEM) for visual characterization of the electronic properties of single-walled carbon nanotubes. VC-SEM involves tuning the electronic band structure and imaging the potential profile along the length of the nanotube. The resultant secondary electron contrast allows to distinguish between metallic and semiconducting carbon nanotubes and to follow the switching of semiconducting nanotube devices, as confirmed by in situ electrical transport measurements. We demonstrate that high-density arrays of individual nanotube devices can be rapidly and simultaneously characterized. A leakage current model in combination with finite element simulations of the device electrostatics is presented in order to explain the observed contrast evolution of the nanotube and surface electrodes. This work serves to fill a void in electronic characterization of molecular device architectures.
\end{abstract}

\section{KEYWORDS}

Carbon nanotubes, electronic properties, voltage-contrast scanning electron microscopy, electrostatics

\section{Introduction}

Single-walled carbon nanotubes (SWCNTs) have tremendous potential as interconnects and field-effect transistors (FETs) in nano-electronics [1, 2]. As this research continues, an increasing demand for rapid, non-invasive electrical characterization techniques becomes apparent. The techniques available so far are direct electron transport measurements and indirect characterization such as Raman spectroscopy [3], near-field optical microscopy [4], scanning tunneling spectroscopy [5] and microscopy [6], electron-diffraction [7] and other scanning probe techniques $[8,9]$. In general, these are slow and sequential, require special substrates or rely on operator skill, and their applicability to the process flow for nanotube device fabrication and integration is limited. Scanning electron microcopy (SEM) could bridge this gap; however, its ability is currently limited to locating and imaging of nanotubes. Here, we establish voltage-contrast scanning electron microscopy (VC-SEM) as an effective metrology tool for the rapid characterization of the electronic nature of SWCNTs and other molecular nanostructures in

Address correspondence to Aravind Vijayaraghavan, v.aravind@int.fzk.de; Ralph Krupke, krupke@int.fzk.de

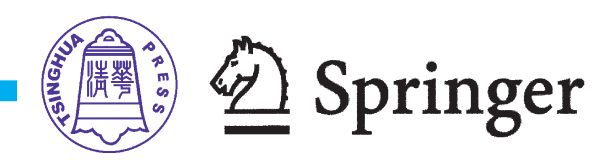


functional device geometries, while overcoming the aforementioned limitations of other techniques. VCSEM involves tuning the electronic band structure and imaging the potential profiles along the length of the nanotube. The results are explained by a leakage current model in combination with finite element simulations of the device electrostatics.

The SEM is based on the detection of secondary electrons (SEs) generated when primary (PE) or backscattered (BSE) electrons interact with the sample [10]. Since the SE yield is proportional to the atomic mass, it is difficult to discern SWCNTs that lie on a silicon dioxide substrate and specific modifications have been proposed in order to enhance the nanotube--substrate contrast. Homma et al. [11] proposed that at a low PE energy of $1.5 \mathrm{keV}$, the insulating substrate adjacent to the nanotube becomes charged with electrons injected from the nanotube and appears brighter than the surrounding substrate. Brintlinger et al. [12] and Zhang et al. [13] used differences in the electron beam-induced selfcharging potential (also at low PE energy of $1 \mathrm{keV}$ ) of the substrate and nanotube to enhance the nanotube contrast. This mechanism is sometimes referred to as voltage contrast (VC) Type 1 [10], and is predominant at low PE energies or under equivalent conditions where the PE penetration depth is smaller than the thickness of the insulating substrate layer. The mechanisms of these contrast enhancement techniques are not definitely established. It has also been shown that such low energy PEs cause severe charging of the substrate and consequently perturb the nanotube's electronic transport properties [14, 15], which is undesirable for nanoelectronic applications. VC-SEM, as described here, involves externally biasing the substrate (sometimes called VC Type 2). VC Types 1 and 2 have been used in microelectronics for failure location in interconnects [16]. An associated mechanism (sometimes called VC Type 3) has been used to image dopant concentrations [17] at inorganic bulk material interfaces. Croitoru et al. [18] and Jesse et al. [19] demonstrated contrast enhancement in multiwall and bundles of singlewall carbon nanotubes, respectively, under external bias conditions, and explained their results in terms of VC Types 2 and 3. However, these methods have not revealed any information about the electronic structure of the nanotubes. Here we develop VCSEM for potential profile imaging and electronic structure characterization in individual molecular nanostructures such as carbon nanotubes as discussed in detail in Section 2. The optimum conditions for VC-SEM, prescribed in Sections 1.3 and 2.1, have been selected such that they minimize the effect of other charging-based contrast mechanisms that do not provide any information about the nanotube electronic structure, but interfere with obtaining effective voltage-contrast images.

\section{Experimental}

\subsection{Nanotube suspensions}

SWCNTs grown by pulsed laser vaporization were dispersed in an aqueous surfactant ( $1 \%$ sodium dodecylbenzene sulfonate) solution followed by ultrasonication and ultracentrifugation to yield a stable suspension of individual SWCNTs. These were fractionated according to length by size-exclusion chromatography and nanotubes with a mean length of $1 \mu \mathrm{m}$ were used for deposition [20, 21].

\subsection{SWCNT device arrays}

High density arrays of single nanotube devices were prepared by low-frequency dielectrophoresis [22] on a highly p-doped Si substrate $(<0.001 \Omega \cdot \mathrm{cm} / \mathrm{sq})$ with an $800 \mathrm{~nm}$ thick thermal $\mathrm{SiO}_{2}$ surface. The deposition technique is self-limiting to one nanotube per electrode pair and the array design allows each nanotube to be characterized individually and independently as a three-terminal device. The electrode pattern was defined by electron beam lithography, followed by metal sputtering and lift-off. The electrodes are composed of a 5-nm Ti adhesion layer and $40 \mathrm{~nm} \mathrm{Pd}$, since Pd has been shown to make low-resistance contact to SWCNTs [23]. Nanotubes were deposited by alternating current (A/C) dielectrophoresis at a peak-to-peak field strength of $2 \mathrm{~V} / \mu \mathrm{m}$ and an A/C frequency of $300 \mathrm{kHz}$. At this low frequency, both metallic and semiconducting nanotubes deposit. Subsequently the devices were annealed at $200{ }^{\circ} \mathrm{C}$ for $2 \mathrm{~h}$ in vacuum to remove residual adsorbed surfactant from the nanotube 
surface and metal-nanotube interface and improve the contact quality.

\subsection{VC-SEM and electrical transport}

The device arrays were mounted in a ceramic package and loaded in a Leo 1530 SEM on a sample holder that allows each lead of the ceramic package to be individually biased. Voltage biasing and transport characterization were performed using an Agilent 4155C Semiconductor Parameter Analyzer. In VC-SEM, the drain is grounded $\left(V_{\mathrm{D}}\right)$, the gate is biased $\left(V_{\mathrm{G}}\right)$, and the source $\left(V_{\mathrm{S}}\right)$ is floating. The gate current is constantly monitored and the device is only imaged after transient currents due to charge redistribution in the oxide under gate bias have subsided ( 1 s). VC-SEM images were obtained by scanning with a $10-\mathrm{keV}$ electron beam of $\sim 2 \mathrm{~nm}$ diameter and $75 \mathrm{pA}$ beam current. The whole array was imaged at 1500X magnification, scanning 2.34 $\times 10^{4} \mu \mathrm{m}^{2}$ in $5.2 \mathrm{~s}$ at a dose of $1.6 \mu \mathrm{C} / \mathrm{cm}^{2}$. To image individual nanotubes, the exposure was limited at $50000 \mathrm{X}$ magnification to a $3 \mu \mathrm{m}^{2}$ area for $800 \mathrm{~ms}$ per scan, resulting in a dose of $2 \mathrm{mC} / \mathrm{cm}^{2}$. SEs were imaged with an annular In-Lens SE detector at $8 \mathrm{~mm}$ working distance. The choice of imaging conditions minimizes the influence of charging-based contrast mechanisms, as described in Section 2.1. For electron transport measurements, to corroborate the VC-SEM observations, in situ tungsten nanoprobes (Picoprobe T-4 series) mounted in Kleindiek Nanotechnik MM3A-EM micromanipulators were used to bias the otherwise floating source electrodes $\left(V_{\mathrm{s}}\right)$ of interest.

\subsection{Simulations}

The finite element simulations presented here were performed with FlexPDE [24], a partial differential equation solver. The central element of the SWCNT FET simulation is a carbon nanotube with a length of $1 \mu \mathrm{m}$ and a radius of $0.63 \mathrm{~nm}$ between two metal electrodes. Both electrodes have a width of $200 \mathrm{~nm}$ and a height of $50 \mathrm{~nm}$. The first electrode is $2 \mu \mathrm{m}$ long and its potential is set to $0 \mathrm{~V}$ (grounded electrode). The other electrode is $1 \mu \mathrm{m}$ long and electrically floating. The full extension of the $x-y$ plane is $5 \times$ $2 \mu \mathrm{m}^{2}$. In the $z$-direction the electrode is separated from the gate electrode at the bottom by an $800 \mathrm{~nm}$ thick oxide layer and from the top electrode by $5 \mu \mathrm{m}$ of air. The potential $(\Phi)$ on the bottom and top surface were set to $V_{\mathrm{G}}$ and $0 \mathrm{~V}$, respectively (Dirichlet condition). Neumann boundary conditions, $\partial \Phi / \partial n$ $=0$, were assigned to the sidewalls of the simulation space as well as to internal material interfaces. The dielectric constants used in this model are $\varepsilon_{\mathrm{r}}=10^{8}$ for the floating metal electrode [25], $\varepsilon_{\mathrm{r}}=3.9$ for the oxide, and $\varepsilon_{\mathrm{r}}=1$ for air. The dielectric constant of the carbon nanotube is varied between $\varepsilon_{\mathrm{r}}=30$ and $\varepsilon_{\mathrm{r}}=$ $10^{8}$. The simulation space is shown in Fig. S-1 (in the Electronic Supplementary Material (ESM)).

To calculate the potential distribution and electric field around a floating or grounded electrode, two separate simulations were performed considering only these electrodes, to reduce computation time. To model the situation for a grounded electrode, the potential of the electrode was also fixed by a Dirichlet condition at $0 \mathrm{~V}$. In the case of the floating electrode, no potential is specified, and the electrode metal was modelled as a dielectric with $\varepsilon_{\mathrm{r}}=10^{8}$ [25]. Due to the large dielectric constant of the metal, the potential of the electrode then adjusts to a constant value within the electrode.

\section{Results and discussion}

\subsection{High density nanotube device arrays}

High density arrays of single nanotube devices [22] (Fig. 1) comprised of independent floating electrodes (source), one common electrode (drain), and the highly-doped $\mathrm{Si} / \mathrm{SiO}_{2}$ substrate as a common back gate were characterized by VC-SEM. Figure 2 shows VC-SEM images of a representative region of the electrode array consisting of 10 nanotube devices, imaged at two illustrative gate voltages $\left(V_{\mathrm{G}}=-10 \mathrm{~V}\right.$ and $+20 \mathrm{~V})$. The devices were maintained at equilibrium condition with the drain grounded $\left(V_{\mathrm{D}}\right.$ $=0 \mathrm{~V}$ ) and source unconnected ( $V_{\mathrm{S}}$ floating), while $V_{\mathrm{G}}$ was swept between 0 and $\pm 20 \mathrm{~V}$. Movies 1 and 2 (in the ESM) show the development of SE contrast for the complete range of these bias conditions. The source electrodes in devices $2,4,7$, and 8 always show identical brightness to the drain for all gate 


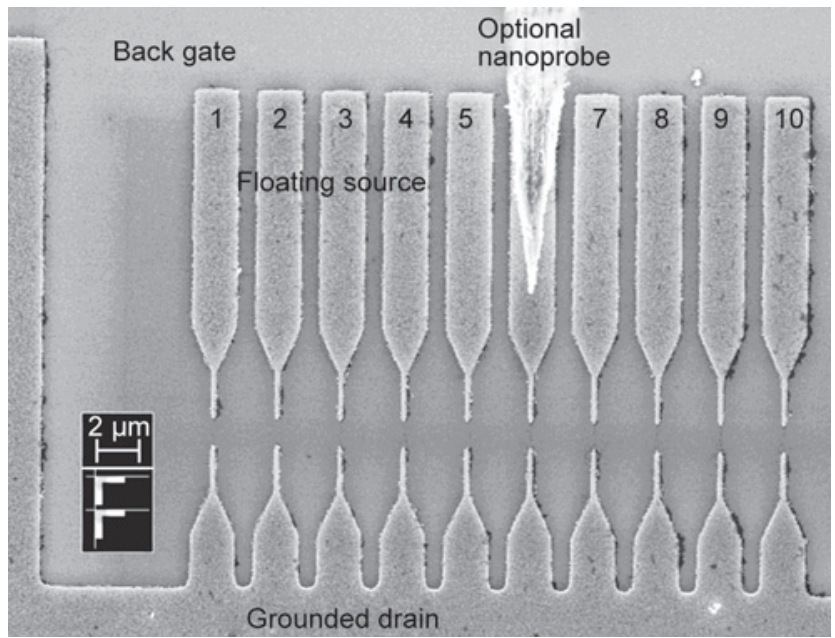

Figure 1 SEM image of a high density single-nanotube device assembly showing 10 three-terminal devices comprising of floating source electrodes $(1, \ldots, 10)$, a drain electrode and a back gate electrode. The drain and back gate electrodes are common to all devices; the drain is grounded $\left(V_{D}=0\right)$, while the gate is biased to $V_{G}$. The source electrodes of all devices are floating $\left(V_{S}\right)$ for voltage-contrast (VC)-SEM studies. In addition, the source electrodes can be explicitly biased by in situ nanoprobes for electrical transport measurements to confirm VC-SEM results. The substrate is composed of $800 \mathrm{~nm} \mathrm{SiO}{ }_{2}$ on top of highly doped silicon. The surface electrodes are composed of $5 \mathrm{~nm} \mathrm{Ti}$ and $40 \mathrm{~nm} \mathrm{Pd}$

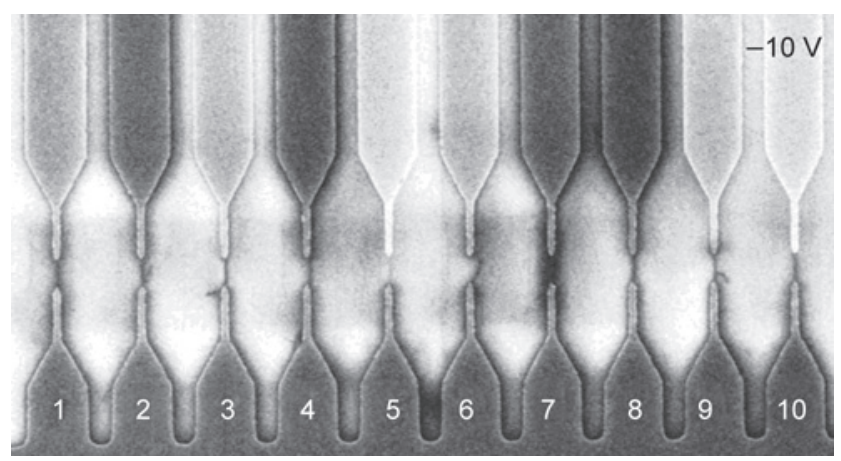

(a)

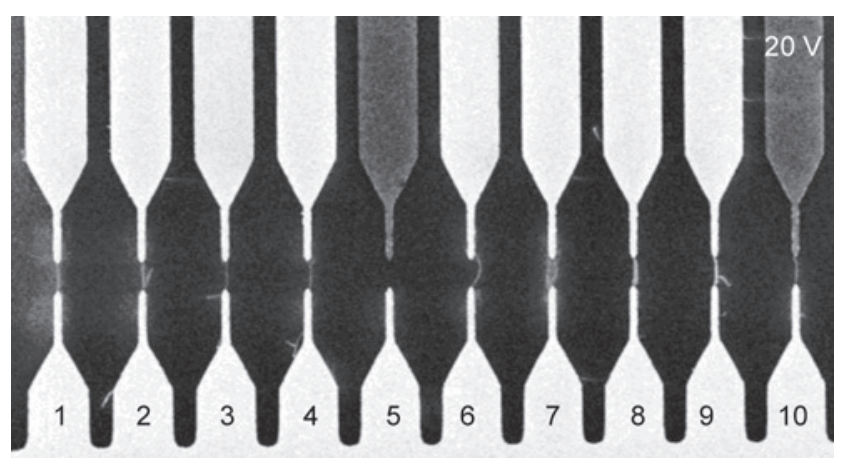

(b)

Figure 2 VC-SEM images of 10 adjacent SWCNT devices at a back gate bias $V_{G}$. (a) $V_{G}=-10 \mathrm{~V}$. Dark contrast indicates suppressed SE emission; (b) $V_{G}=+20 \mathrm{~V}$. Bright contrast indicates enhanced SE emission. The common drain (bottom electrode) is grounded and the independent sources (top electrodes) are floating. Devices 2, 4, 7 , and 8 are metallic; devices $1,3,6$, and 9 are semiconducting voltages, indicating that the potential between source and drain equilibrates instantaneously through the bridging nanotube. These nanotube devices are metallic. The brightness of the source electrodes of devices 1, 3, 6, and 9 lag significantly behind the drain contrast because, at low $V_{\mathrm{G}}$, the potential does not equilibrate between the source and drain via the bridging nanotube. The devices behave similarly to metallic devices at high $V_{\mathrm{G}}$. These are semiconducting nanotube devices. Source electrodes of devices 5 and 10 do not change their brightness with applied bias even though there is a nanotube in the gap. Closer observation reveals that in device 5 the nanotube does not make effective contact with the drain while in device 10 the contact to the source is broken. These observations were confirmed by measuring the electron transport characteristics of the three-terminal devices (Figs. 3 (c) and (d)) with the floating electrode contacted by an in situ nano-probe. The data shows that the brightness of the floating electrode, which can be rapidly imaged at low resolution, correlates to the electronic property of the corresponding nanotube device.

For an interpretation of our VC-SEM images, it is necessary to know the origin of SEs that we are detecting in our experiment and their energy distribution. The In-Lens SE detector used in our SEM detects only low energy SE1 electrons [26], which are a result of the direct impact of the PE with the substrate, and responsible for voltage-contrast. The Everhart-Thornley (SE2) detector, mounted outside the column, primarily collects higher energy SE2, SE3, and backscattered electrons which are not affected by surface potentials and therefore unsuitable for VCSEM. Indeed, we have experimentally confirmed that no voltage contrast can be observed if the SE2 detector is used. SE yield depends strongly on the surface potential which augments the material work function. SEs have low energy $(\leqslant 50 \mathrm{eV})$ compared to the incident beam (1-30 keV) and only those originating at depths up to a few nanometers from the surface can escape and be detected. When the surface has a negative potential (negative $V_{\mathrm{G}}$ ), the SE emission yield is enhanced while a positive potential (positive $V_{\mathrm{G}}$ ) effectively retains the SEs and reduces their yield [27]. This is equivalent to shifting the SE energy spectrum 


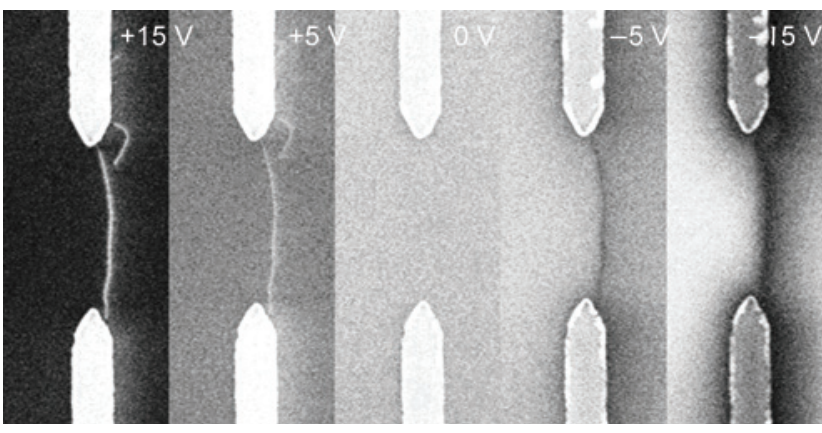

(a)

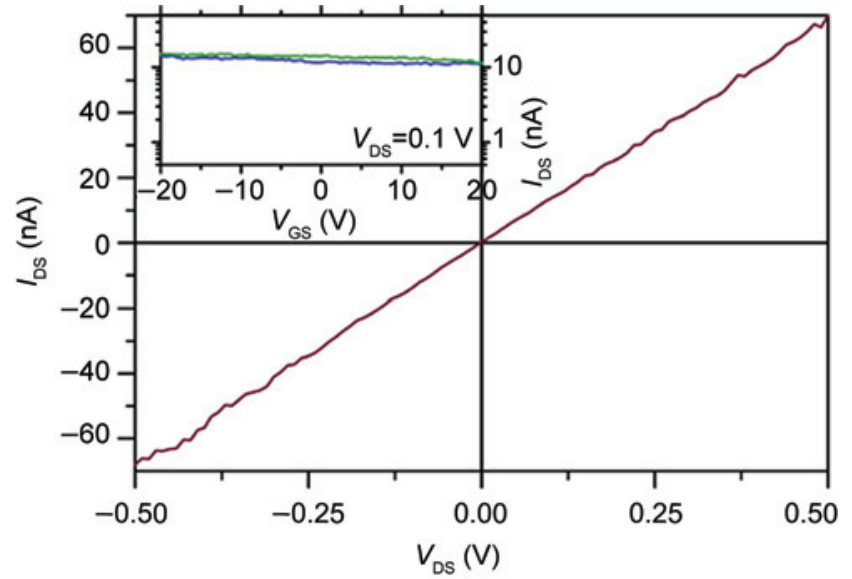

(c)

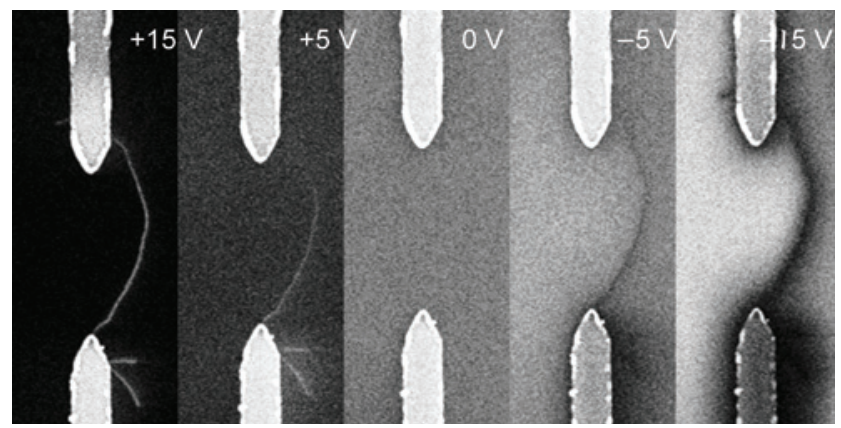

(b)

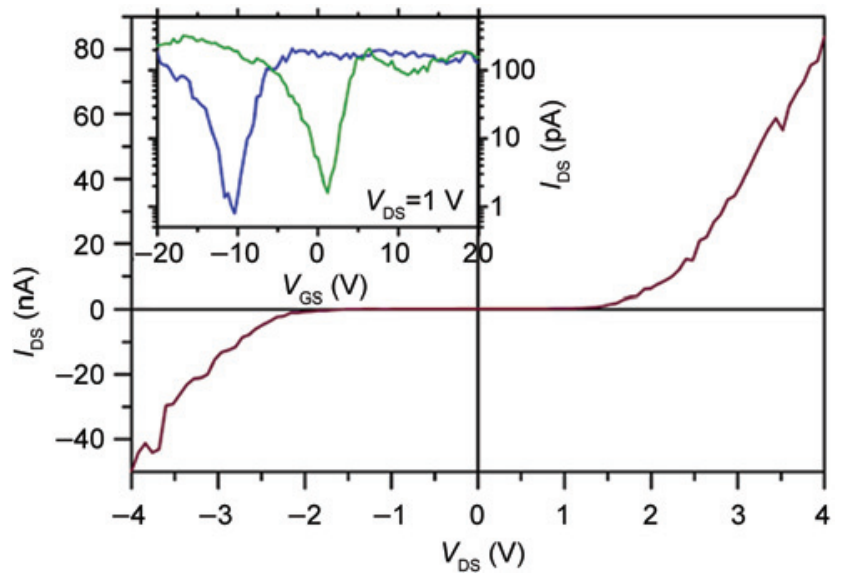

(d)

Figure 3 Contrast profile under selected back gate bias conditions $V_{G}=0 V_{1} \pm 5 V_{1} \pm 15 V$ in a (a) metallic and (b) semiconducting SWCNT. Metallic nanotubes uniformly acquire the same contrast as the drain (uniform potential distribution) and instantaneously equilibrates the contrast of the source and the drain. Semiconducting nanotubes have a non-uniform contrast (non-uniform potential distribution) at low bias conditions and the source contrast equilibrates only at high bias. Nanotubes generally not discernable at high scan speeds ( $0 \mathrm{~V}$ images) become clearly visible under biased conditions. The metallic or semiconducting nature is confirmed through in situ three-terminal transport measurements. The red curve shows $I_{D S}-V_{D S}$, and the blue and green curves are the forward and reverse sweep of the $I_{D S}-V_{G}$, respectively. (c) Metallic nanotubes have linear $I_{D S}-V_{D S}$ characteristics and $V_{G}$ has no influence on their conductance (inset). (d) Semiconducting nanotubes have nonlinear $I_{D S}-V_{D S}$ and their conductance changes over orders of magnitude in response to $V_{G}$ (inset). In this case, the SWCNT FET is ambipolar

towards higher or lower energies respectively. Any potential on an SWCNT or electrode will influence SE image contrast both due to changes in the SE yield (VC Type 2) and the deflection of SEs emitted from the substrate in the vicinity of the nanotube or electrode by transverse electric fields (VC Type 3) [10, 17].

In this framework, the contrast evolution of the electrodes can be understood as follows. Surface electrodes of a nanotube device are either grounded drain electrodes $\left(V_{\mathrm{D}}\right)$ or floating source electrodes $\left(V_{\mathrm{S}}\right)$. The potential of a floating source electrode is determined by the leakage current $\left(I_{\text {leak }}\right)$ between the source and gate electrode through the gate oxide, and by the ratio of the gate oxide resistance $\left(R_{\mathrm{OX}}\right)$ to the nanotube resistance $\left(R_{\mathrm{NT}}\right) . R_{\mathrm{OX}}$ depends on $V_{\mathrm{S}}$ $V_{\mathrm{G}}$, and $R_{\mathrm{NT}}$ depends on $V_{\mathrm{G}}$ and $V_{\mathrm{S}}-V_{\mathrm{D}}$. If $R_{\mathrm{OX}}\left(V_{\mathrm{S}}-\right.$ $\left.V_{\mathrm{G}}\right)<R_{\mathrm{NT}}\left(V_{\mathrm{S}}-V_{\mathrm{D}}, V_{\mathrm{G}}\right)$, then $V_{\mathrm{S}} \approx V_{\mathrm{G}}$ and if $R_{\mathrm{OX}}\left(V_{\mathrm{S}}-\right.$
$\left.V_{\mathrm{G}}\right)>R_{\mathrm{NT}}\left(V_{\mathrm{S}}-V_{\mathrm{D}}, V_{\mathrm{G}}\right)$, then $V_{\mathrm{S}} \approx V_{\mathrm{D}}$. In the case of a metallic nanotube device $R_{\mathrm{OX}} \gg R_{\mathrm{NT}}$ and $V_{\mathrm{S}}=V_{\mathrm{D}}$, hence the source and drain electrodes appear with similar brightness independent of $V_{\mathrm{G}}$. In the case of a defective nanotube or nonbridged device $R_{\mathrm{OX}} \ll R_{\mathrm{NT}}$ and $V_{\mathrm{S}}=V_{\mathrm{G}}$, hence the source and drain electrodes appear with different brightness at any gate voltage. In the case of a semiconducting nanotube device, two regimes can be distinguished: For small $V_{\mathrm{G}}, R_{\mathrm{OX}}$ $<R_{\mathrm{NT}}$ and $V_{\mathrm{S}} \approx V_{\mathrm{G}}$, and at large $V_{\mathrm{G}}, R_{\mathrm{OX}}>R_{\mathrm{NT}}$ and $V_{\mathrm{S}}$ $\approx V_{\mathrm{D}}$. Hence, with increasing $V_{\mathrm{G}}$ the brightness of the source and drain electrodes deviate significantly before approaching similar values. We derived $V_{\mathrm{S}}$ for a typical semiconducting nanotube device by comparing the brightness of the floating source electrode with the brightness of the drain electrode $\left(V_{\mathrm{D}}\right.$ $=0)$ and a non-bridged floating electrode $\left(V_{\mathrm{S}}^{0}=V_{\mathrm{G}}\right)$.

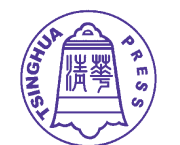


Figure 4 shows how $V_{\mathrm{S}}$ increases with $V_{\mathrm{G}}$ up to $V_{\mathrm{G}} \approx$ $\pm 8 \mathrm{~V}$ and then decreases until $V_{\mathrm{S}} \approx V_{\mathrm{D}}$ at $V_{\mathrm{G}}= \pm 20 \mathrm{~V}$. The $V_{\mathrm{S}}$ vs $V_{\mathrm{G}}$ dependence is symmetric in $V_{\mathrm{G}}$, which provides evidence that the leakage current $I_{\text {leak }}$ is not electron beam-induced as in conventional electron beam-induced current imaging (EBIC). In fact, we constantly monitor the current through the drain electrode, which in the steady-state is always below our detection limit of $100 \mathrm{fA}$. We have estimated that $I_{\text {leak }} \approx 10^{-15}$ A at $V_{\mathrm{G}} \approx 10 \mathrm{~V}$ on the basis of the bulk resistivity of $\mathrm{SiO}_{2}$, the lateral source electrode

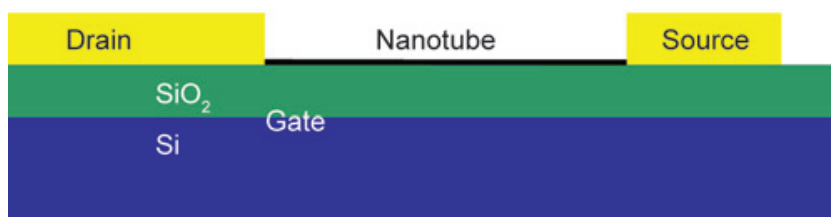

(a)

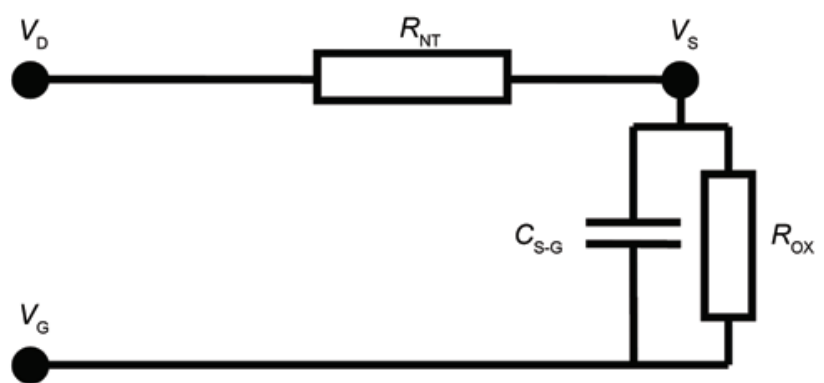

(b)

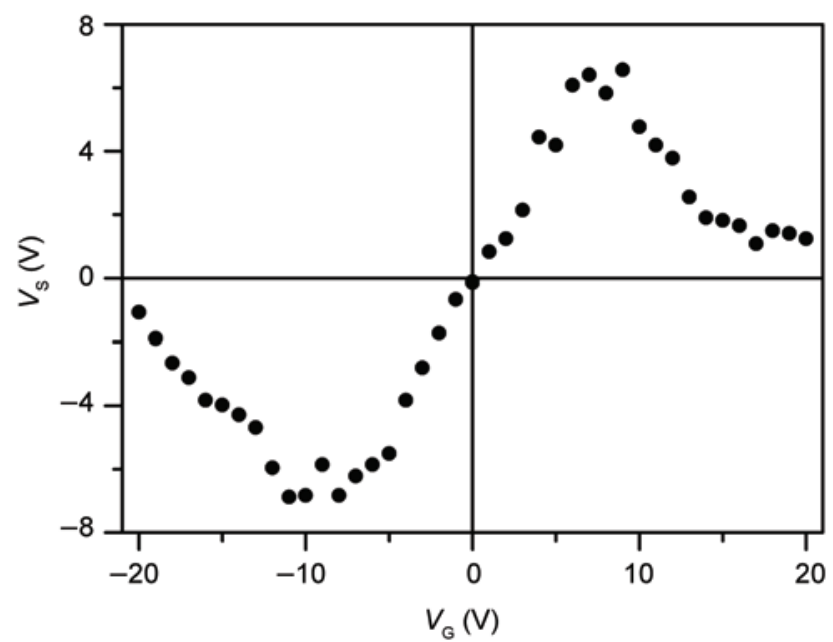

(c)

Figure 4 (a) Schematic cross-section of one of the devices; (b) equivalent circuit diagram with the nanotube resistance $R_{\mathrm{NT}}$, the oxide resistance $R_{\mathrm{Ox}}$ and the source electrode capacitance $C_{\mathrm{S}-\mathrm{G}}$; (c) floating source potential $V_{S}$ of a semiconducting device vs gate potential $V_{G}$, obtained by comparison of the brightness of the floating source electrode with the brightness of the drain electrode $\left(V_{D}=0\right)$ and $a$ non-bridged floating electrode $\left(V_{S}^{0}=V_{G}\right)$ dimension and the gate oxide thickness. This sets the resistance to $10^{16} \Omega$ at which $V_{\mathrm{S}}$ acquires half the value of $V_{\mathrm{G}}$. Note that this resistance in combination with the floating source electrode capacitance of $C \approx 10^{-16} \mathrm{~F}$ also sets the characteristic time scale for the transient regime to approximately 1 s. In Fig. S-2 (in the ESM) we show the simulated equipotential lines and electric field distribution around a floating surface electrode with $V_{\mathrm{S}}=V_{\mathrm{G}}$ and a grounded surface electrode with $V_{\mathrm{S}}=V_{\mathrm{D}}=0$, as an example for $V_{\mathrm{G}}= \pm$ $5 \mathrm{~V}$. The local electric fields on top and at the edges of the electrodes are responsible for VC Type 2 and VC Type 3, respectively, where VC Type 2 determines the electrode brightness and VC Type 3 causes brightness variations at the electrode edges.

It should be noted that there exist some subtle differences between probing the electronic structure of a nanotube by VC-SEM or by transconductance measurements. In VC-SEM, the floating electrode bridged by a semiconducting nanotube always equilibrates with the grounded electrode potential and contrast at similar positive and negative gate bias, an indication of ambipolar electronic characteristics. In electronic transport measurements however, the devices were either ambipolar or p-type unipolar with very low or moderate ONstate conductance at positive $V_{\mathrm{G}}$. Our proposed explanation for this difference is as follows. The unipolarity in electrical transport measurements of large band gap semiconducting SWCNT devices is an effect of the metal-nanotube Schottky barrier at the contact [28]. In the case of Pd electrodes, the metal Fermi level is aligned close to the conduction band and, the band-bending at the contacts is asymmetrical; large for positive and small for negative gate bias. Large band-bending results in a small ON-state conductance due to the low tunneling transmission probability; therefore, such devices are p-type unipolar. For contacts where the metal Fermi level is aligned close to the valence band, a n-type unipolar device is expected by similar arguments. All nanotubes are however intrinsically ambipolar and capable of transmitting electrons and holes with equal effectiveness [29]. Metallic nanotubes often make poor contact with the electrodes, either due to intrinsic weak electronic coupling at the Fermi 
surface [30] between the nanotube and metal or extrinsic factors such as an intervening surfactant layer [22]. This results in a large contact resistance and low overall device conductance, which is not representative of the intrinsic conductivity $[23,31]$ and electronic properties of an SWCNT. In VC-SEM, only a very small current flows through the nanotube under all conditions, limited either by the nanotube or oxide resistance under different conditions. Apparently, it probes the intrinsic ambipolarity of all semiconducting nanotubes and is insensitive to the contact Schottky barriers. The current limitation by contact resistance seems not to affect VCSEM measurements in the way it affects electrical transport measurements, and VC-SEM can be used to characterize nanotube devices irrespective of the effectiveness of the metal-nanotube contact. Further evidence for this statement will be provided in another publication.

To conclude this section, we note that VC Type 1 due to charging is minimized in our experiment by the primary electron penetration depth of 1.5 $\mu \mathrm{m}$ at $10 \mathrm{keV}[32,33]$ being significantly larger than the oxide thickness of $800 \mathrm{~nm}$. Most of the primary electrons pass through the oxide into the silicon gate and are removed by the gate bias circuit. They do not contribute to the accumulation of negative charge in the substrate. On the other hand, the secondary electron and backscattered electron yields of $\mathrm{Si}$ and $\mathrm{SiO}_{2}$ are similar. The bias on the gate is not enough to affect the yield of the highenergy backscattered electrons, and since secondary electrons are only released from a small surface layer of few nanometers, their yield is also not affected by the underlying conducting substrate. As a result prolonged exposure to electron irradiation, under the VC-SEM conditions presented here, results in an overall positive charging of the substrate and a darkoffset in the contrast of all features, unlike the negative charging expected for thick insulating substrates. This dark-offset is also enhanced by the deposition of an organic layer on the substrate surface under the influence of the electron beam. The time- and dosescales for these effects are however at least an order of magnitude larger than what is required for VCSEM and their effects can thus be neglected here. A low PE energy of $1 \mathrm{keV}$ results in a penetration of only $30 \mathrm{~nm}$, and causes large negative charging of the substrate, which is undesirable.

\subsection{Individual nanotube devices}

The evolution of potential and brightness in the floating electrode is a measure of the overall characteristics of the device. Devices might exhibit semiconducting characteristics due to various reasons: the bridging nanotube is semiconducting, a Stone-Wales defect [34] or electron irradiationinduced transformation [14], or impurity-induced rectifying behavior [35]. However, at a higher magnification, VC-SEM can also image the voltage profile within the nanotube itself, and provide information about the electronic structure of an individual SWCNT.

Figures 3(a) and (b) show the contrast along the nanotubes of a metallic (device 4) and semiconducting (device 6) device, under illustrative gate bias conditions $\left(V_{\mathrm{G}}=0 \mathrm{~V}, \pm 5 \mathrm{~V}, \pm 15 \mathrm{~V}\right)$. The complete sequence $\left(V_{\mathrm{G}}=0 \mathrm{~V}\right.$ to $\left.\pm 20 \mathrm{~V}\right)$ is presented in Movies 3 to 6 (in the ESM). It is apparent that at different $V_{\mathrm{G}}$, the contrast along a metallic nanotube remains uniform, while it is non-uniform along the length of a semiconducting nanotube until very high $V_{\mathrm{G}}$. This behavior corresponds to the difference in contrast of the floating source electrode in metallic and semiconducting devices. The contrast profile reflects the voltage profile along the length of the nanotube. Metallic SWCNTs have no gap in their electronic density of states and their conductance is unaffected by a gate field. Therefore, the mobile carriers can effectively equilibrate the potential along the nanotube under all bias conditions. Semiconducting SWCNTs have a band gap of up to $1 \mathrm{eV}$ depending on their chirality and diameter, and their conductance is strongly influenced by the bending of their electronic band structure by a gate field. Also important to note is the "glow" around the nanotube; this is a result of the local in-plane electric fields (VC Type 3) (Fig. 5).

To conclude, VC-SEM can probe the potential distribution along a carbon nanotube in a similar way to scanning probe techniques like electrostatic force microscopy [9] or sliding contact measurements [8]. However, the acquisition times for VC-SEM are 


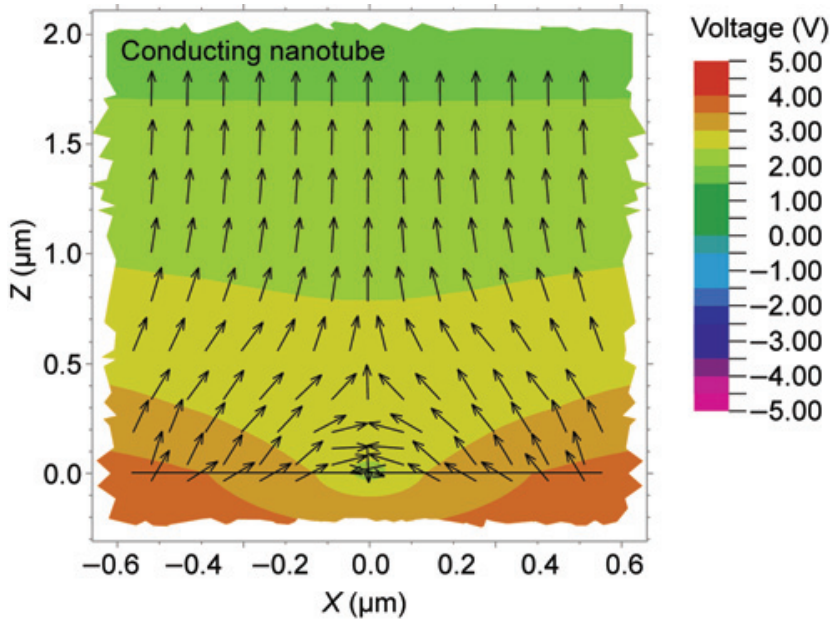

(a)

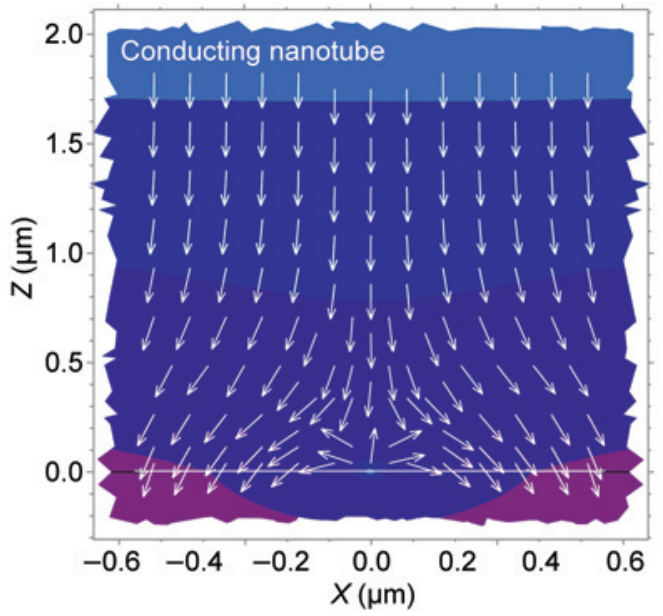

(b)

Figure 5 Electrostatic potential $\Phi$ (background color) and electric field $E$ (arrows indicate direction only) distribution around a conducting nanotube, connected to the grounded drain, in a crosssection perpendicular to its axis. Electrons experience a force opposite to the direction of $E$ : (a) positive gate bias, nanotube appears bright; (b) negative gate bias, nanotube appears dark. The transverse components to the electric field are responsible for the observed "glow" around the nanotubes

orders of magnitude shorter than for scanning probe techniques, and there is no need to explicitly bias each source electrode.

\subsection{Electrostatic modeling}

A quantitative model of VC-SEM would require simulation of the SE generation and propagation under the influence of the potential and electric field distribution around the nanoelectronic device. On the other hand, a qualitative understanding of the SE contrast can be obtained from the surface potential and electric field distribution around the nanotube and associated electrodes. The local potential $(\Phi)$ could be derived by solving self-consistently the Poisson-Schrödinger equation [36] as a function of $V_{S}$, $V_{\mathrm{D}}$, and $V_{\mathrm{G}}$, including electron-phonon scattering and hot carriers in the system. However, this approach is beyond the scope of this paper. Therefore, we limit the discussion to solutions of the Laplace equation $(\nabla(\varepsilon \nabla \Phi)=0)$, which gives valid results for metallic nanotube devices $\left(V_{\mathrm{S}}=V_{\mathrm{D}}, \varepsilon=10^{8} \varepsilon_{0}\right)$ and for semiconducting nanotube devices in the subthreshold regime $\left(V_{\mathrm{S}}=V_{\mathrm{G}}, \varepsilon=30 \varepsilon_{0}\right)$. The threshold regime of a semiconducting nanotube device has been roughly approximated by variation of $\varepsilon$.

Figure 6 shows the calculated potential distribution in the vicinity of the nanotube and surface electrodes for different nanotube permittivity. While the simulations were performed at $V_{\mathrm{G}}=5 \mathrm{~V}$ and $V_{\mathrm{D}}$ $=0$, the potential distributions scale proportionally for other values of $V_{\mathrm{G}}$. We note that within the simulations the floating electrode potential $V_{\mathrm{S}}$ is determined by the electrostatics while experimentally $V_{\mathrm{S}}$ is determined by $R_{\mathrm{Ox}}$ and $R_{\mathrm{NT}}$. Nevertheless the simulations reproduce qualitatively the experimental $V C-S E M$ results. When a semiconducting SWCNT is insulating $\left(\varepsilon=30 \varepsilon_{0}\right)$, then $V_{\mathrm{S}}=V_{\mathrm{G}}$ and the

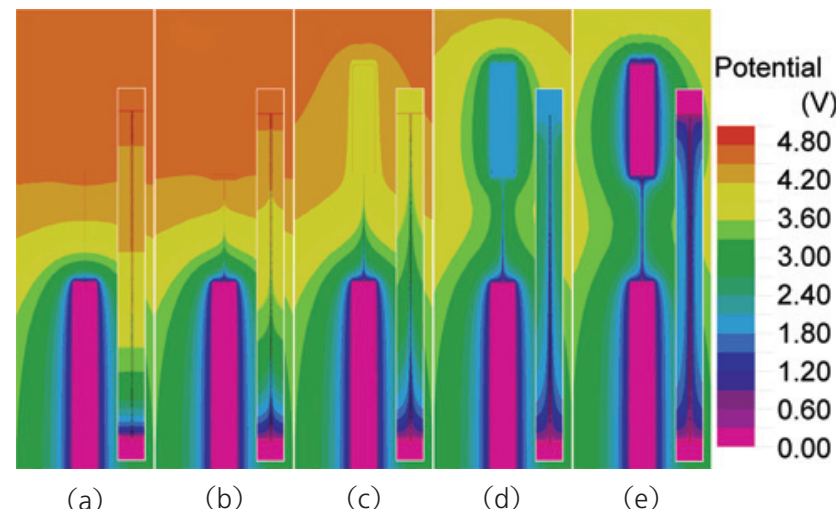

Figure 6 Electrostatic potential $(\Phi)$ evolution as a function of the dielectric constant $\varepsilon_{\mathrm{r}}$ of the bridging nanotube. (a)-(e) $\varepsilon_{\mathrm{r}}=30$, $10^{5}, 10^{6}, 10^{7}$, and $10^{8}$. Insets show the magnified region around the nanotube and the potential profile along the nanotube. The simulations were performed at $V_{G}=5 \mathrm{~V} . V_{D}=0 . V_{S}$ is floating. The potentials are scalable for other values of $V_{G}$. (a) $\left(\varepsilon_{r}=30\right)$ corresponds to a semiconducting nanotube at low gate bias, completely in its OFF state, with $V_{\mathrm{S}}=V_{\mathrm{G}}$ since $R_{\mathrm{OX}}<R_{\mathrm{NT}}$ (see text). (e) $\left(\varepsilon_{\mathrm{r}}=10^{8}\right)$ corresponds to either a semiconducting nanotube at high gate bias (ON state) or a metallic nanotube, with $V_{\mathrm{S}}=V_{\mathrm{D}}$ since $R_{\mathrm{OX}}>R_{\mathrm{NT}}$. Intermediate values of $\varepsilon_{\mathrm{r}}$ mimic the transition from OFF to ON state of the semiconducting nanotube under increasing gate bias 
brightness of the floating electrode is similar to the substrate. As the SWCNT becomes more conducting, mimicked here by $\varepsilon>30 \varepsilon_{0}$, the potential of the floating electrode and its brightness start to increasingly deviate from the substrate. When a semiconducting SWCNT is in its ON state, or for a metallic SWCNT $\left(\varepsilon=10^{8} \varepsilon_{0}\right)$, then $V_{\mathrm{S}}=V_{\mathrm{D}}$ and the brightness of the floating source electrode is similar to that of the drain and distinct from the substrate. In this case, the nanotube has the same potential as the electrodes (and hence uniform contrast) along its length. For the intermediate regime, the potential and contrast along the nanotube decrease from the drain to the source.

The simulations reproduce the experimental results only qualitatively due to the limitations of the underlying model. The experimental SE contrast and the simulated potential distribution are compared in Fig. 7 (and Figs. S-3 and S-4 in the

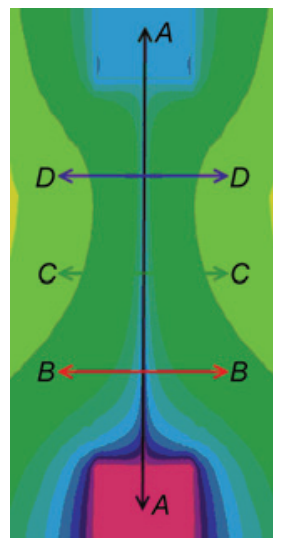

(a)

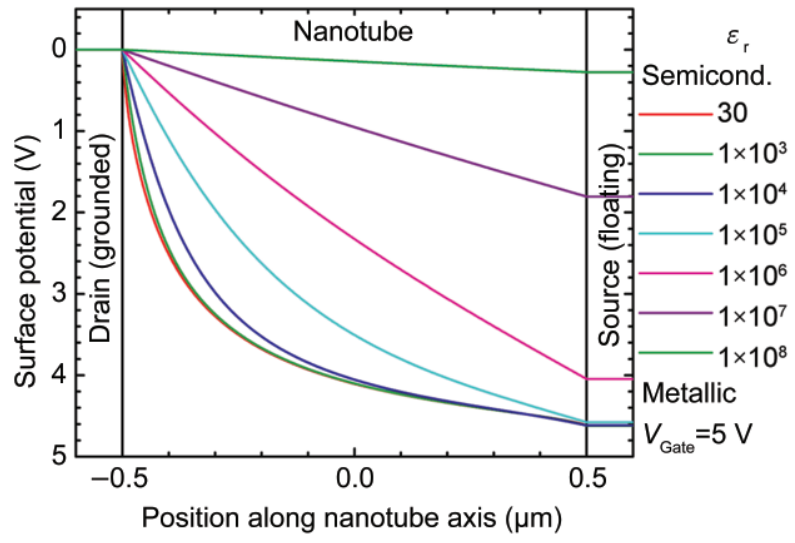

(b)

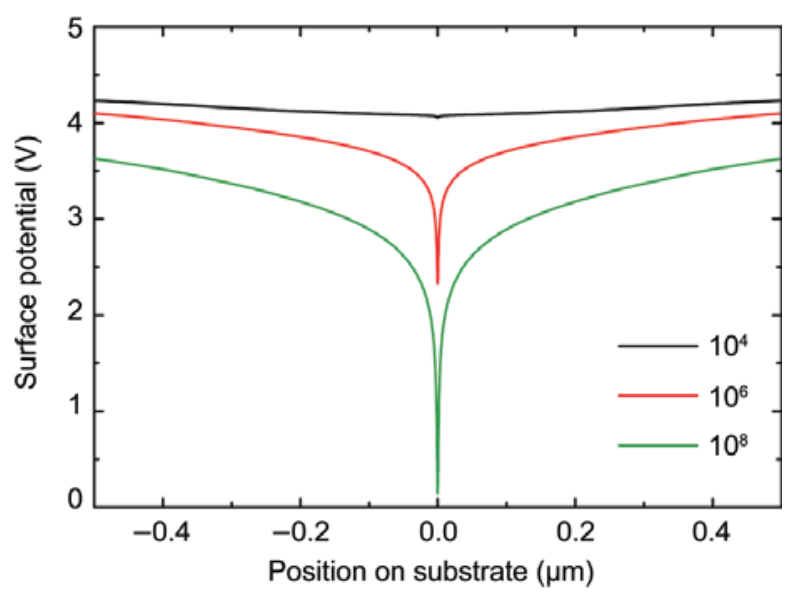

(c)

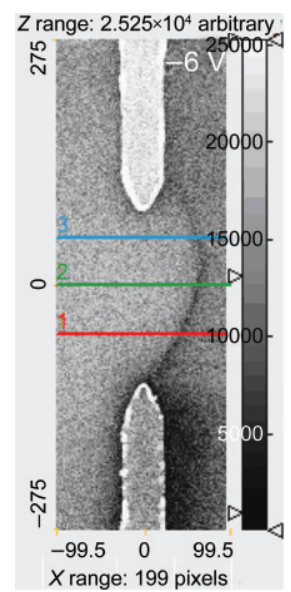

(d)

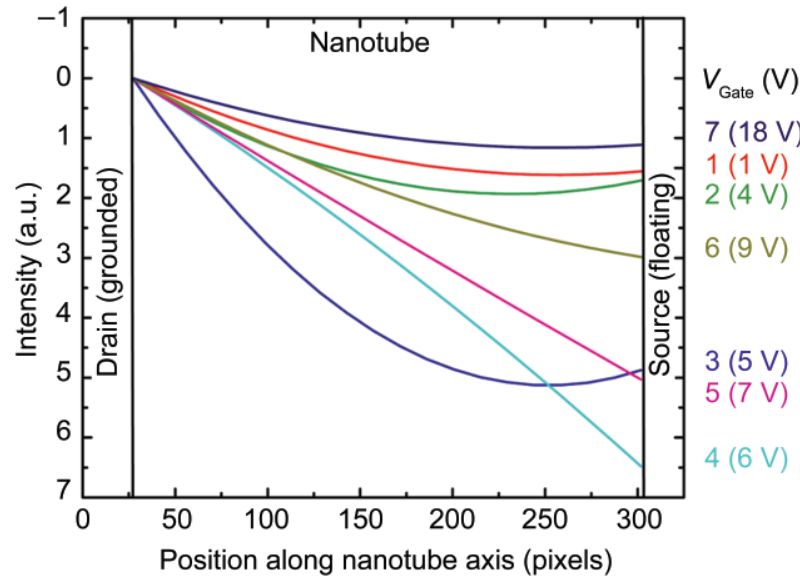

(e)

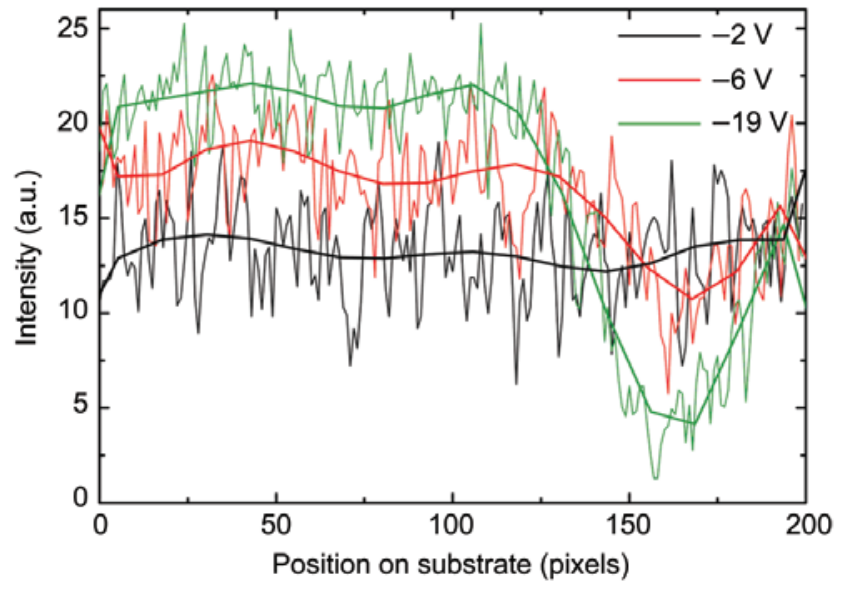

( $f$ )

Figure 7 Comparison between numerical simulations and experimental observations. The cross-sections of interest are shown for (a)

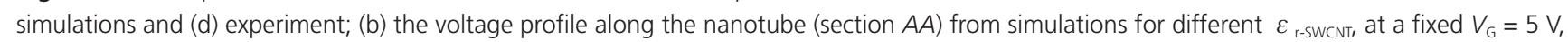
which can be scaled for other values of $V_{G}$ i (c) the variation of surface potential across the nanotube along section CC at three different values of $\varepsilon_{\text {r-SWCNT }}$; (e) polynomial fits to the experimental contrast profile along the nanotube for different values of $V_{G}$ (raw data in the ESM); ( $f$ ) the experimental contrast profile (and polynomial fit) across the middle of the nanotube for three values of $V_{G}$. The experimental contrast around the nanotube is broader because of the deflections of SEs around the nanotube by transverse components of the electric field. Further comparison at various cross-sections is shown in the ESM

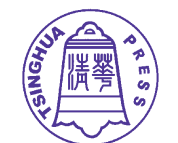


ESM). Simulation indicates that the potential drops off rapidly on either side of the nanotube, however, the electrons are deflected by the transverse electric field (Fig. 5), resulting in the broader contrast "glow" observed experimentally around the nanotube. Along the length of the nanotube, the simulations approximate the observed experimental profile. The full width at half maximum of the SE energy distribution from $\mathrm{SiO}_{2}$ is $\sim 2 \mathrm{~V}$; therefore, for surface potential shifts greater than a few volts, the contrast saturates accordingly and does not follow the potential distribution.

\subsection{Advantages of VC-SEM}

In contrast to competing techniques, VC-SEM is based on SEM, which is a user-friendly and routine characterization tool for SWCNTs. It works effectively on any substrate of interest to the nanoelectronics community. Nanotube devices can be characterized rapidly and simultaneously, which is of particular importance as SWCNT electronics reaches integration densities comparable to siliconbased microelectronics. VC-SEM can serve as a routine characterization technique in SWCNT device fabrication to efficiently sift through a large number of individual nanotubes to locate those which are suitable or interesting for further analysis with complementary techniques. Such a characterization tool is an essential advancement in realizing the full potential of SWCNTs in commercial applications [37].

The enhanced contrast of a nanotube under bias in an SEM allows it to be imaged at much lower magnifications and higher scan speeds and observed more clearly at comparable magnifications. By imaging the contrast of the source, we can identify metallic and semiconducting nanotube devices at even lower magnification and resolution where the nanotube itself is indiscernible. This allows for the simultaneous and rapid electrical characterization of a vast, high density array of SWCNT devices [22]. These results are particularly useful, since prolonged exposure to electron irradiation could have detrimental effects on nanotube devices [14] and VCSEM can significantly reduce the imaging time and exposure effects.

\section{Conclusions}

This work serves to establish VC-SEM as a new and versatile method to characterize the electronic properties of SWCNTs in device configurations, particularly, the ability to distinguish metallic and semiconducting SWCNTs in an SEM. Metallic and semiconducting SWCNTs and their devices show differences in contrast due to distinct potential distributions acquired by the nanotube and the floating electrodes under gate bias which can be understood within a leakage current model. In order to observe the best VC-SEM results, certain conditions should be met: (1) SE1 electrons, which are sensitive to surface potentials, are preferentially detected over SE2, SE3, and backscattered electrons; (2) surface-potential under external bias shifts the SE1 energy distribution (VC Type 2) and lateral electric fields deflect emerging the SE1 electrons; (3) charging (VC Type 1) and EBIC contrast mechanisms are suppressed.

In summary, VC-SEM can probe the potential distribution along a carbon nanotube, providing information about its electronic structure. It can be extended to characterize defects and discontinuities (device failure) in nanotubes. Systematic analysis of the contrast profiles and verification by complementary single nanotube photoluminescence mapping could reveal correlations to the electronic band-gap of semiconducting SWCNTs and enable us to distinguish different nanotube chiralities in an SEM. VC-SEM can be similarly used to characterize other nanoelectronic materials such as organic FETs, bio-electronic systems and low-dimensional materials like graphene.

\section{Acknowledgements}

The authors acknowledge Ferdinand Evers, Matthias Hettler and David John for helpful discussions. The research was funded by the Initiative and Networking Fund of the Helmholtz-Gemeinschaft Deutscher Forschungszentren and equipment grant from Agilent Technologies.

Electronic Supplementary Material: Figures S-1-S-4 and Movies 1-6 are available in the online version of 
this article at http://dx.doi.org/10.1007/s12274-

008-8034-3 and are accessible free of charge.

\section{References}

[1] McEuen, P. L. Single-wall carbon nanotubes. Phys. World 2000, 13, 31-36.

[2] Saito, R.; Fujita, M.; Dresselhaus, G.; Dresselhaus, M. S. Electronic-structure of graphene tubules based on $C_{60}$. Phys. Rev. B 1992, 46, 1804-1811.

[3] Oron-Carl, M.; Hennrich, F.; Kappes, M. M.; Lohneysen, H. V.; Krupke, R. On the electron-phonon coupling of individual single-walled carbon nanotubes. Nano Lett. 2005, 5, 1761-1767.

[4] Qian, H. H.; Gokus, T.; Anderson, N.; Novotny, L.; Meixner, A. J.; Hartschuh, A. Near-field imaging and spectroscopy of electronic states in single-walled carbon nanotubes. Phys. Status Solidi B 2006, 243, 3146-3150.

[5] Venema, L. C.; Janssen, J. W.; Buitelaar, M. R.; Wildoer, J. W. G.; Lemay, S. G.; Kouwenhoven, L. P.; Dekker, C. Spatially resolved scanning tunneling spectroscopy on single-walled carbon nanotubes. Phys. Rev. B 2000, 62, 5238-5244.

[6] Wildoer, J. W. G.; Venema, L. C.; Rinzler, A. G.; Smalley, R. E.; Dekker, C. Electronic structure of atomically resolved carbon nanotubes. Nature 1998, 391, 59-62.

[7] Jiang, H.; Nasibulin, A. G.; Brown, D. P.; Kauppinen, E. I. Unambiguous atomic structural determination of singlewalled carbon nanotubes by electron diffraction. Carbon 2007, 45, 662-667.

[8] Yaish, Y.; Park, J. Y.; Rosenblatt, S.; Sazonova, V.; Brink, M.; McEuen, P. L. Electrical nanoprobing of semiconducting carbon nanotubes using an atomic force microscope. Phys. Rev. Lett. 2004, 92, 046401.

[9] Bachtold, A.; Fuhrer, M. S.; Plyasunov, S.; Forero, M.; Anderson, E. H.; Zettl, A.; McEuen, P. L. Scanned probe microscopy of electronic transport in carbon nanotubes. Phys. Rev. Lett. 2000, 84, 6082-6085.

[10] Seiler, H. Secondary electron emission in the scanning electron microscope. J. Appl. Phys. 1983, 54, R1-R18.

[11] Homma, Y.; Suzuki, S.; Kobayashi, Y.; Nagase, M.; Takagi, D. Mechanism of bright selective imaging of singlewalled carbon nanotubes on insulators by scanning electron microscopy. Appl. Phys. Lett. 2004, 84, 17501752.
[12] Brintlinger, T.; Chen, Y. F.; Durkop, T.; Cobas, E.; Fuhrer, M. S.; Barry, J. D.; Melngailis, J. Rapid imaging of nanotubes on insulating substrates. Appl. Phys. Lett. 2002, 81, 2454-2456.

[13] Zhang, R. Y.; Wei, Y.; Nagahara, L. A.; Amlani, I.; Tsui, $\mathrm{R}$. K. The contrast mechanism in low voltage scanning electron microscopy of single-walled carbon nanotubes. Nanotechnology 2006, 17, 272-276.

[14] Vijayaraghavan, A.; Kanzaki, K.; Suzuki, S.; Kobayashi, Y.; Inokawa, H.; Ono, Y.; Kar, S.; Ajayan, P. M. Metalsemiconductor transition in single-walled carbon nanotubes induced by low-energy electron irradiation. Nano Lett. 2005, 5, 1575-1579.

[15] Marquardt, C. W.; Dehm, S.; Vijayaraghavan, A.; Blatt, S.; Hennrich, F.; Krupke, R. Reversible metal-insulator transitions in metallic single-walled carbon nanotubes. Nano Lett. 2008, 8, 2767-2772.

[16] Pfeiffer, H. C.; Langner, G. O.; Stickel, W.; Simpson, R. A. Contactless electrical testing of large area specimens using electron beams. J. Vac. Sci. Tech. 1981, 19, 10141018.

[17] Buzzo, M.; Ciappa, M.; Millan, J.; Godignon, P.; Fichtner, W. Two-dimensional dopant imaging of silicon carbide devices by secondary electron potential contrast. Microelectron. Eng. 2007, 84, 413-418.

[18] Croitoru, M. D.; Bertsche, G.; Kern, D. P.; Burkhardt, C.; Bauerdick, S.; Sahakalkan, S.; Roth, S. Visualization and in situ contacting of carbon nanotubes in a scanning electron microscope. J. Vac. Sci. Tech. B 2005, 23, 27892792.

[19] Jesse, S.; Guillorn, M. A.; Ivanov, I. N.; Puretzky, A. A.; Howe, J. Y.; Britt, P. F.; Geohegan, D. B. In situ electricfield-induced contrast imaging of electronic transport pathways in nanotube-polymer composites. Appl. Phys. Lett. 2006, 89, 013114

[20] Hennrich, F.; Krupke, R.; Lebedkin, S.; Arnold, K.; Fischer, R.; Resasco, D. E.; Kappes, M. Raman spectroscopy of individual single-walled carbon nanotubes from various sources. J. Phys. Chem. B 2005, 109, 10567-10573.

[21] Arnold, K.; Hennrich, F.; Krupke, R.; Lebedkin, S.; Kappes, M. M. Length separation studies of single walled carbon nanotube dispersions. Phys. Status Solidi B 2006, 243, 3073-3076.

[22] Vijayaraghavan, A.; Blatt, S.; Weissenberger, D.; OronCarl, M.; Hennrich, F.; Gerthsen, D.; Hahn, H.; Krupke, 
R. Ultra-large-scale directed assembly of single-walled carbon nanotube devices. Nano Lett. 2007, 7, 15561560.

[23] Mann, D.; Javey, A.; Kong, J.; Wang, Q.; Dai, H. J. Ballistic transport in metallic nanotubes with reliable $\mathrm{Pd}$ ohmic contacts. Nano Lett. 2003, 3, 1541-1544.

[24] www.pdesolutions.com.

[25] Konrad, A.; Graovac, M. The finite element modeling of conductors and floating potentials. IEEE T. Magn. 1996, 32, 4329-4331.

[26] Jaksch, H. Zeiss NTS GmbH. Private communication.

[27] Wells, O. C. Scanning electron microscopy; McGraw-Hill: New York, 1974.

[28] Martel, R.; Derycke, V.; Lavoie, C.; Appenzeller, J.; Chan, K. K.; Tersoff, J.; Avouris, P. Ambipolar electrical transport in semiconducting single-wall carbon nanotubes. Phys. Rev. Lett. 2001, 87, 256805.

[29] Jarillo-Herrero, P.; Sapmaz, S.; Dekker, C.; Kouwenhoven, L. P.; van der Zant, H. S. J. Electron-hole symmetry in a semiconducting carbon nanotube quantum dot. Nature 2004, 429, 389-392.

[30] Tersoff, J. Contact resistance of carbon nanotubes. Appl.
Phys. Lett. 1999, 74, 2122-2124.

[31] White, C. T.; Todorov, T. N. Carbon nanotubes as long ballistic conductors. Nature 1998, 393, 240-242.

[32] Krupke, R.; Malik, S.; Weber, H. B.; Hampe, O.; Kappes, M. M.; von Lohneysen, $H$. Patterning and visualizing selfassembled monolayers with low-energy electrons. Nano Lett. 2002, 2, 1161-1164.

[33] Kanaya, K.; Okayama, S. Penetration and energy-loss theory of electrons in solid targets. J. Phys. D: Appl. Phys. 1972, 5, 43-58.

[34] Anderson, N.; Hartschuh, A.; Novotny, L. Chirality changes in carbon nanotubes studied with near-field Raman spectroscopy. Nano Lett. 2007, 7, 577-582.

[35] Antonov, R. D.; Johnson, A. T. Subband population in a single-wall carbon nanotube diode. Phys. Rev. Lett. 1999, 83, 3274-3276.

[36] John, D. L.; Castro, L. C.; Pereira, P. J. S.; Pulfrey, D. L. A Schrödinger-Poisson solver for modeling carbon nanotube FETs. Nanotechnology 2004, 3, 65-68.

[37] Baughman, R. H.; Zakhidov, A. A.; de Heer, W. A. Carbon nanotubes - The route toward applications. Science 2002, 297, 787-792. 